\title{
Development of Fast Disintegrating Compressed Tablets Using Amino Acid as Disintegratation Accelerator: Evaluation of Wetting and Disintegration of Tablet on the Basis of Surface Free Energy
}

\author{
Jinichi Fukami, ${ }^{a}$ Asuka Ozawa, ${ }^{b}$ Yasuo Yoshinashi, ${ }^{b}$ Etsuo Yonemochi, ${ }^{b}$ and Katsuhide TeradA ${ }^{*}, b$ \\ ${ }^{a}$ Kyoto Pharmaceutical Industries, Ltd.; 38 Nishinokyo, Tukinowa-cho, Nakagyo-ku, Kyoto 604-8444, Japan: and \\ ${ }^{b}$ Faculty of Pharmaceutical Sciences, Toho University; 2-2-1 Miyama, Funabashi, Chiba 274-8510, Japan. \\ Received June 7, 2005; accepted September 5, 2005
}

A fast disintegrating compressed tablet was formulated using amino acids, such as L-lysine HCl, L-alanine, glycine and L-tyrosine as disintegration accelerator. The tablets having the hardness of about $\mathbf{4}$ kgf were prepared and the effect of amino acids on the wetting time and disintegration time in the oral cavity of tablets was examined on the basis of surface free energy of amino acids. The wetting time of the tablets increased in the order of L-lysine $\mathrm{HCl}$, L-alanine, glycine and L-tyrosine, whereas the disintegration time in the oral cavity of the tablets increased in the order of L-alanine, glycine, L-lysine $\mathrm{HCl}$ and L-tyrosine. These behaviors were well analyzed by the introduction of surface free energy. When the polar component of amino acid was large value or the dispersion component was small value, faster wetting of tablet was observed. When the dispersion component of amino acid was large value or the dispersion component was small value, faster disintegration of tablet was observed, expect of L-tyrosine tablet. The fast disintegration of tablets was explained by the theory presented by Matsumaru.

Key words fast disintegrating tablets; amino acid; surface free energy; solubility

With an increasing interest of new oral dosage forms for the geriatrics and pediatric patients, many researches have been performed. ${ }^{1-10)}$ In many dosage forms for elderly patients, fast disintegrating tablets in oral cavity have intrigued pharmaceutical researchers. Fast disintegrating tablets can be an interesting oral dosage forms for geriatric and pediatric patients because of their difficulties of swallowing of conventional tablets. To disintegrate fast in oral cavity, researchers developed them for focusing on giving tablets with porous structure. ${ }^{11)}$ There are some methods to give tablets with porous structures, those are to lyophilize charging in preformed blister pockets ${ }^{12)}$ or to mold or compress ${ }^{13)}$ wet powders containing drugs using sugar or sugar alcohols as main excipients. However, specific facilities and techniques are required for the manufacturing of fast disintegrating tablets.

In this study, we attempted to prepare the fast disintegrating compressed tablets using amino acids with ordinary pharmaceutical processes, since the amino acid such as glycine was used as disintegratation accelerator. ${ }^{14)}$ Terada and Yonemochi. ${ }^{15)}$ reported that the change of the surface free energy of talc by the grinding was the effect on the rapid disintegration time of tablet and the faster dissolution rate of ethenzamide from tablet. Then, the disintegration mechanism for fast disintegrating tablets has been discussed from the viewpoint of surface free energy etc.

\section{Experimental}

Materials Glycine and hydroxypropylcellulose-L (HPC-L) were supplied by Kyoto Pharmaceutical Industries, Ltd. L-Alanine (Musasino Chemical Laboratory, Ltd.), L-tyrosine (Tokyo Kasei Kogyo Co., Ltd.), L-lysine $\mathrm{HCl}$ (Ajinomoto Co., Ltd.), cornstarch (Kozakai Pharmaceutical Co., Ltd.) and magnesium stearate (Wako Pure Chemical Industries, Ltd.) were purchased. Table 1 presents the formulation for the preparation of tablet.

To evaluate the surface free energy of amino acids, following solvents were used.

Benzene (Nacalai Tesque, Inc.), $n$-hexane (Wako Pure Chemical Industries, Ltd.), nitroethane (Tokyo Kasei Kogyo Co., Ltd.), 1-octanol, toluene (Nakarai Tesque, Inc.), carbon tetrachloride (Wako Pure Chemical Industries, Ltd.), cyclopentanol (Tokyo Kasei Kogyo Co., Ltd.), benzyl alcohol
(Wako Pure Chemical Industries, Ltd.), formamide (Tokyo Kasei Kogyo Co., Ltd.), methanol (Wako Pure Chemical Industries, Ltd.) and 1,5-pentanediol (Tokyo Kasei Kogyo Co., Ltd.) were purchased. The reagents with $99.5 \%$ or more of purity were used with out further purification.

Preparation of Granules Amino acid and cornstarch were passed through a 100 mesh sieve. Eighteen point one gram of amino acid and $6.5 \mathrm{~g}$ of the cornstarch were mixed in a V-type mixer (Tsutsui Rikagaku Kikai Co., Ltd.) for $10 \mathrm{~min}$. After mixing of the powders, 5\% HPC-L aq. solution of $4 \mathrm{ml}$ as binder was gradually added to the mixture, in quantity sufficient to achieve the state of agglomeration. Mixing was ceased after the addition of $5 \%$ HPC-L aq. solution and the wet mass of about $25 \mathrm{~g}$ was allowed to pass through a 16 mesh sieve with pressing by the hand. The passing wet granules were dried in an oven at $60^{\circ} \mathrm{C}$ for about $30 \mathrm{~min}$, then left to cool down at room temperature. The granules of the $30-100$ mesh sieve fraction were obtained.

Compression Magnesium stearate passed through a 100 mesh sieve was added to the granules before compression. The granules were compressed with flat-faced punches, in a die of $7.0 \mathrm{~mm}$ internal diameter using a hydraulic press and tablets $(250 \mathrm{mg})$ were prepared, which have the hardness of about $4 \mathrm{kgf}$. In order to obtain tablets of the same hardness, the granules of L-lysine $\mathrm{HCl}$, L-alanine, glycine and L-tyrosine were compressed under 500, 1500,5000 and $1500 \mathrm{kgf} / \mathrm{cm}^{2}$, respectively. The hardness of tablets was measured with the tablet hardness tester of Monsanto type. The result of hardness for tablets was shown in Fig. 1. The tablets with hardness of $4 \mathrm{kgf}$ were obtained. The thickness of tablets of L-lysine $\mathrm{HCl}$, L-alanine, glycine and Ltyrosine were $0.4816,0.4025,0.374$ and $0.4052 \mathrm{~cm}$, respectively.

Wetting Time The wetting time of tablet was measured by the method described by Bi et al. ${ }^{16)}$ The method is as follows. A piece of paper tissue folded twice was placed in a small culture dish, and the time for complete wetting was measured at $25^{\circ} \mathrm{C}$. The data of the wetting time were used the

Table 1. Formulation of Fast Disintegration Tablets

\begin{tabular}{lrrrr}
\hline \hline & $\begin{array}{c}\text { L-Lysine } \\
\text { HCl tablet }\end{array}$ & $\begin{array}{c}\text { L-Alanine } \\
\text { tablet }\end{array}$ & $\begin{array}{c}\text { Glycine } \\
\text { tablet }\end{array}$ & $\begin{array}{c}\text { L-Tyrosine } \\
\text { tablet }\end{array}$ \\
\hline L-Lysine HCl (mg) & 181 & 0 & 0 & 0 \\
L-Alanine (mg) & 0 & 181 & 0 & 0 \\
Glycine (mg) & 0 & 0 & 181 & 0 \\
L-Tyrosine (mg) & 0 & 0 & 0 & 181 \\
Corn starch (mg) & 65 & 65 & 65 & 65 \\
HPC-L (mg) & 2 & 2 & 0 & 2 \\
Magnesium stearate (mg) & 2 & 2 & 0 & 2 \\
\hline
\end{tabular}




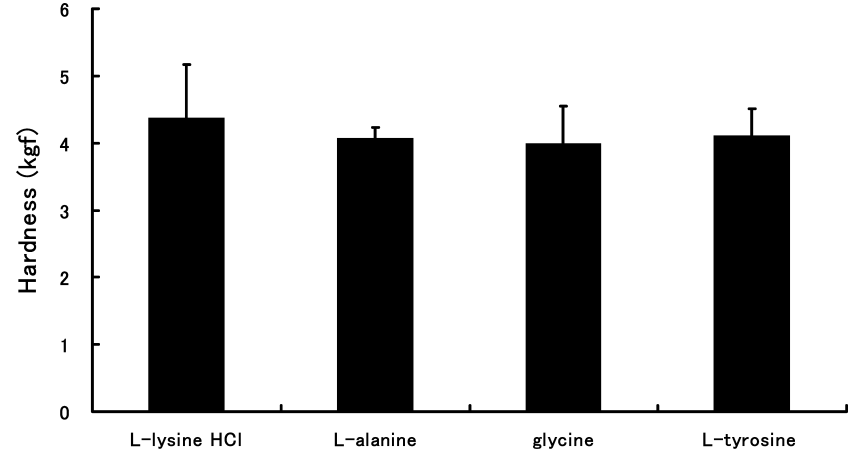

Fig. 1. The Hardness of Tablets Prepared at the Constant Hardness

mean of ten measurements.

Disintegration Time in the Oral Cavity Measurements of disintegration time in the oral cavity were carried out in 5 healthy volunteers (mean age $=22$ ), who were randomly administered four kinds of tablets at $24 \mathrm{~h}$ intervals. After the mouth was rinsed with purified water, one tablet was held in the mouth until the tablet disintegrated without chewing. The disintegration time was recorded with seconds.

Evaluation of Surface Free Energy of Amino Acids A given quantity of powder was introduced in the glass tube, and the tube was then tapped until the height of the powder bed became constant. The liquid penetration rate of the powder bed was measured by using KRUSS K12 (KRUSS $\mathrm{GmbH})$ at $25^{\circ} \mathrm{C}$. The solutions with which the value of the surface free energy is known were used in order to evaluate surface free energy of amino acids. The glass tube packed with powder was lowered into liquid, and time zero was recorded when the liquid contacted to the powder. The weight of liquid penetrated into the powder bed was recorded against time. The penetration rate constants for various liquids were calculated by the Washburn equation. ${ }^{17)}$ The data of the penetration rate constants were used the mean of five measurements for each liquids.

The contact angle between liquid and powder was evaluated from the penetration rate constants. The surface free energy of amino acids was evaluated from Owens, Wendt, Rable, and Kaelble's equation (1) using the values of the contact angle and the known surface free energy of liquids.

$$
\gamma_{\mathrm{L}} \cdot(1+\cos \theta)=2 \sqrt{\gamma_{\mathrm{S}}^{\mathrm{d}} \gamma_{\mathrm{L}}^{\mathrm{d}}}+2 \sqrt{\gamma_{\mathrm{S}}^{\mathrm{p}} \gamma_{\mathrm{L}}^{\mathrm{p}}}
$$

where $\theta$ is contact angle, $\gamma_{\mathrm{L}}$ and $\gamma_{\mathrm{S}}$ are the surface free energy of the liquid and the solid respectively and $\mathrm{p}$ and $\mathrm{d}$ represent polar and dispersion component of the surface free energy, respectively. From Eq. 1, polar and dispersion component of the solid are obtained.

\section{Result and Discussion}

Effect of Amino Acids on the Wetting Time of Tablets The wetting time of tablets with amino acids was examined by the liquid penetration method. The wetting time of each tablet is shown in Fig. 2. The wetting time of tablets was differed significantly dependent on the kinds of amino acids used for the formulations.

The wetting time was short in the order of L-lysine $\mathrm{HCl}$, Lalanine, glycine and L-tyrosine. Especially, the wetting time of tablet with L-tyrosine was significantly slow to finish the whole wetting compared with those of other amino acids (the wetting time of tablet prepared by L-tyrosine was 26.8 times slower than that of L-lysine $\mathrm{HCl}$ ). It is usually acquired that the penetration of water into tablet is depends on the solubility of amino acids. To know whether the solubility of amino acids affects the wetting of tablet, the solubility of amino acids to water was examined. The solubility of amino acids to water is found to be $73.0 \mathrm{~g} / 100 \mathrm{~g}$ for L-lysine $\left.\mathrm{HCl},{ }^{18}\right)$ $25.0 \mathrm{~g} / 100 \mathrm{~g}$ for glycine, ${ }^{19)} 16.7 \mathrm{~g} / 100 \mathrm{~g}$ for L-alanine ${ }^{19)}$ and $0.105 \mathrm{~g} / 100 \mathrm{~g}$ for L-tyrosine ${ }^{19)}$ at $25^{\circ} \mathrm{C}$. It was found that the solubility of these amino acids was significantly dispersed.

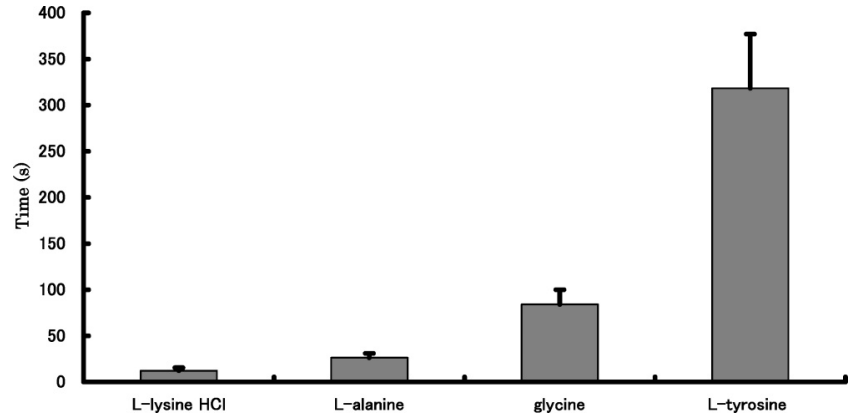

Fig. 2. Effect of Amino Acids on the Wetting Time of Tablets

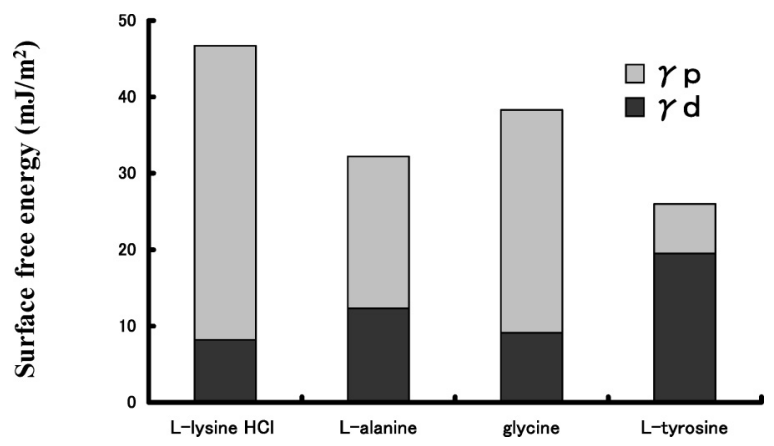

Fig. 3. Comparison of Polar and Dispersion Component of Surface Free Energy of Amino Acids

The wetting time has a tendency to become small as the solubility of amino acid rises. So, the solubility of amino acid to water affects the wetting of tablet, which is partly due to the ability of water penetration into capillary of tablet.

Estimation of Wetting Properties on the Basis of Surface Free Energy of Amino Acids The first step of disintegration behavior is wetting of surface of the powder beds. The wetting time of the tablets prepared by various amino acids have been changed with the amino acid. It seemed that this difference was a difference of physical properties of the amino acid and the wetting is directly related to the surface property. So, the surface free energy of amino acids was estimated using solvent penetration methods. Figure 3 shows the dispersion component and polar component of surface free energy of each amino acid.

Each amino acid has different dispersion and polar surface free energy. L-Lysine $\mathrm{HCl}$ has a larger polar surface free energy, on the other hand, L-tyrosine has a larger dispersion surface free energy. In case of the wetting of amino acid to water, more polar amino acid has a stronger affinity to water because water is regarded as a polar solvent. Figure 4 shows the relationship between the wetting time and polar component or dispersion component of amino acids used for the formulations.

Almost linear tendency was observed between the wetting time of tablets and the polar component of amino acids, that is, a fast wetting of tablet was observed when a more polar amino acid was used. As the same reason mentioned above, a reverse tendency was observed between the wetting time of tablets and the dispersion component of amino acid, that is, a slow wetting of tablet was observed when a less polar amino acid was used for the formulation.

Effect of Amino Acids on the Disintegration Time in 


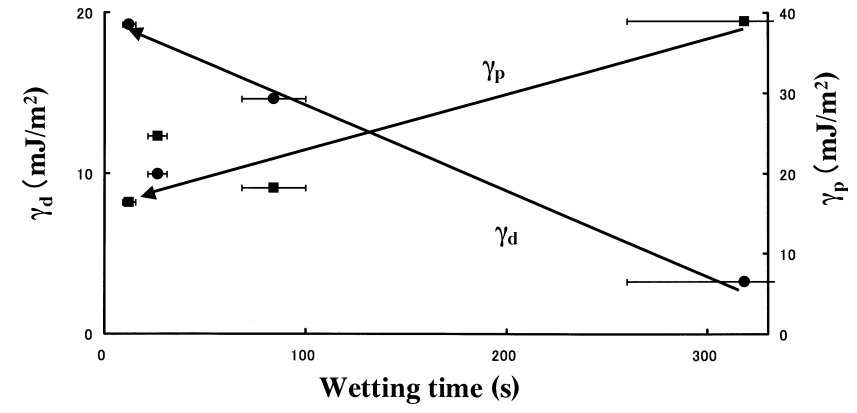

Fig. 4. Relationship between $\gamma_{\mathrm{p}}, \gamma_{\mathrm{d}}$ of Amino Acids and the Wetting Time

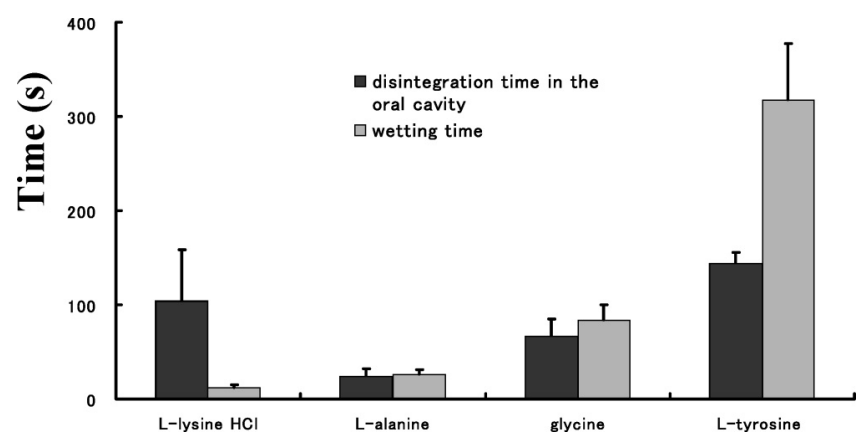

Fig. 5. Relationship between the Wetting Time and Disintegration Time in the Oral Cavity of the Tables

Oral Cavity It is usually considered that the disintegration time of oral cavity directly relate to the wetting time of water into tablet. So, the disintegration time of tablet in oral cavity was examined using healthy volunteers. Figure 5 shows the relation between the disintegration time in oral cavity and the wetting time of tables for these amino acids.

For the tablets with L-lysine $\mathrm{HCl}$, although the wetting time of tablet was fairly fast, however, the disintegration time in oral cavity was considerably slow anticipated from the wetting time. As a whole, it was found that there was no direct correlation between disintegration time in oral cavity and wetting time of tablets for these amino acids. The surface free energy was plotted against the disintegration time of tablet. Completely different tendency was observed between the disintegration time and surface property of amino acids as shown in Fig. 6.

The amino acid with less polar surface free energy showed faster disintegration except for L-tyrosine.

Influence of Surface Free Energy of Amino Acids on the Disintegration Time in the Oral Cavity According to the theory proposed by Matsumaru, ${ }^{20)}$ the force separating the two crystalline faces acts on the outside along these surfaces when the water penetrates into tablet between hydrophobic and hydrophilic faces. Yoshihashi et al. ${ }^{21)}$ reported that the disintegration rate of tablet was increased when phenytoin contents have increased in the microcrystallinephenytoin two components formulation studied by microcalorimetry. This is the supporting experimental result of Matsumaru's theory that the force works to separate the interface of phenytoin and microcrystalline cellulose when the water penetrates into tablet, because phenytoin is hydrophobic compound and microcrystalline cellulose is hydrophilic compound. Then the disintegration of tablet has occurred fast

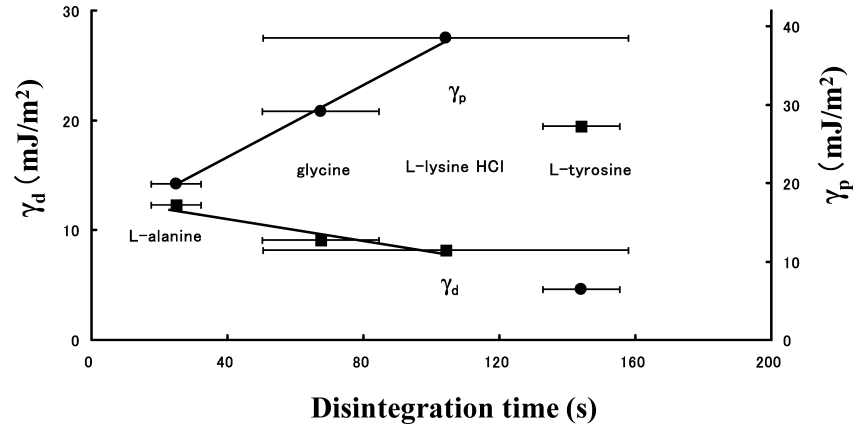

Fig. 6. Relationship between the Disintegration Time in the Oral Cavity and $\gamma_{\mathrm{p}}, \gamma_{\mathrm{d}}$ of Amino Acids

when phenytoin content was increased. The formulation of tablet mainly consists of amino acid and cornstarch, latter has a hydrophilic properties. So, the formulation of hydrophobic amino acid and hydrophilic cornstarch would work better disintegration. In this study, neither polar nor dispersion component of surface free energy of L-tyrosine were related to disintegrating time in oral cavity. For disintegration of tablets, it is necessary that tablet was wetting and water was penetrated into tablets. The wetting of L-tyrosine tablet slowed considerably slower than the wetting of other tablets. For this reason, the disintegration of tablet became slow, too. However, the value of disintegrating time/wetting time was the smaller than that of other tablets. Then, it is thought that the disintegrating time of L-tyrosine tablet does not contradict the theory of Matsumaru. Therefore, it was thought that the polar component and dispersion component of surface free energy of amino acid was greatly related to the disintegration time in oral cavity.

\section{Conclusion}

A fast disintegrating tablet was formulated using amino acids, such as L-lysine $\mathrm{HCl}$, L-alanine, glycine and L-tyrosine. The wetting time of the tablets increased in the order of L-lysine $\mathrm{HCl}$, L-alanine, glycine and L-tyrosine, whereas the disintegration time in the oral cavity of the tablets increased in the order of L-alanine, glycine, L-lysine $\mathrm{HCl}$ and L-tyrosine. These behaviors were well analyzed by the introduction of surface free energy. When the polar component of amino acid was large value, the fast wetting time of tablet was observed. When the dispersion component of amino acid was large value, the faster disintegration of tablet was observed. It was found that major factor affecting disintegration of tablet was high dispersion component as well as low polar component of surface free energy of amino acids.

\section{References}

1) Abdelbary G., Eouani C., Prinderre P., Joachim J., Reynier J., Piccerelle P., Int. J. Pharm., 292, 29 - 41 (2005).

2) Thomas S., Ulrich F. S., Helmut L., J. Pharm. Sci., 94, 120-133 (2005).

3) Narazaki R., Harada T., Takami N., Kato Y., Ohwaki T., Chem. Pharm. Bull., 52, 704-707 (2004).

4) Shimizu T., Nakano Y., Morimoto S., Tabata T., Hamaguchi N., Igari Y., Chem. Pharm. Bull., 51, 942-947 (2004).

5) Simone S., Peter C. S., Eur. J. Pharm. Sciences, 15, 295-305 (2002).

6) Jin Y., Ohkuma H., Wang H., Natsume H., Sugibayashi K., Morimoto Y., Yakuzaigaku, 122, 989-994 (2002).

7) Johannes C., Jean S., Faiz A., Frances K., Diane B., Clin. Ther, 22, 1035-1048 (2000).

8) Watanabe A., Hanawa T., Sugihara M., Yakuzaigaku, 54, 77-87 
(1994).

9) Hanawa T., Tsuchiya T., Ikoma R., Hidaka M., Sugihara M., Chem. Pharm. Bull., 43, $284-288$ (1995).

10) Corveleyn S., Remon J. P., Int. J. Pharm., 152, $215-225$ (1997).

11) Ichikawa T., Mukai B., Shiraishi S., Utoguchi N., Fujii M., Matsumoto M., Watanabe Y., Chem. Pharm. Bull., 49, 134-139 (2001).

12) Masaki K., The collected papers of the 22nd Conference on Pharmaceutical Technology; Academy of Pharmaceutical Science and Technology, Japan, Tokyo, 1997, pp. 79-84.

13) Tsushima Y., Farumashia, 33, 1119-1123 (1997).

14) Liu H., Zhuanli Shenqing Gongkai Shuomingshu, 2004, 15 (2004).
15) Terada K., Yonemochi E., Solid State Ionics, 172, 459-462 (2004).

16) Bi Y., Sunada H., Yonezawa Y., Danjo K., Otsuka A., Iida K., Chem. Pharm. Bull., 44, 2121-2127 (1996).

17) Washburn E. W., Phys. Rev., 17, 273-283 (1921).

18) Ajinomoto Co., Inc., "Amino Acid Handbook," 1st ed., Kogyo Chosakai Publishing Inc. Press, Tokyo, 2003, pp. 127-129.

19) “The Merck Index," 13th ed., Merck Research Laboratories Division of Merck, Whitehouse Station, N.J., 2001.

20) Matsumaru H., Yakugaku Zasshi, 79, 854-855 (1959).

21) Yoshihashi Y., Makita M., Yamamura S., Fukuoka E., Terada K., Chem. Pharm. Bull., 46, 437-477 (1998). 